\title{
Aplicabilidade do Sistema de Classificação da Função Motora Grossa (GMFCS) na paralisia cerebral - revisão da literatura
}

\author{
Applicability of the classification system of gross motor function (GMFCS) in
} cerebral palsy - review

Ana Ivone Antonia de Oliveira', Marina Ortega Golin', Márcia Cristina Bauer Cunha'

\begin{abstract}
Resumo
A quantidade de métodos de avaliação padronizados aplicáveis a crianças com Paralisia Cerebral é reduzida. 0 Sistema de Classificação da Função Motora Grossa (GMFCS) é um dos instrumentos mais utilizados na clínica e na pesquisa, mundialmente. 0 objetivo deste artigo é realizar revisão bibliográfica sobre a aplicabilidade do Sistema de Classificação da Função Motora Grossa (GMFCS) na Paralisia Cerebral. 0 campo de busca incluiu consulta via Bireme de base de dados eletrônicos: Lilacs, Medline, PubMed e Scielo, assim como livros, monografias e teses relacionadas. Esta pesquisa foi realizada no CADIP (Centro de Aprendizagem, Documentação, Informação e Pesquisa da FMABC, Santo André). Os dados deste estudo podem ser úteis na caracterização funcional do quadro clínico, no direcionamento para escolha de avaliações e no planejamento das intervenções clínicas a essa população. 0 GMFCS é uma ferramenta simples, porém considerada essencial pela literatura consultada, pois ao permitir a detecção exata do potencial motor da criança possibilita maior eficácia terapêutica e maior homogeneidade nas pesquisas.
\end{abstract}

Palavras-chave: Paralisia cerebral; transtornos das habilidades motoras; destreza motora.

\section{Abstract}

The quantity of standardized assessment methods applicable to children with Cerebral Palsy is reduced. The Gross Motor Function Classification System (GMFCS) is one of the most used rating systems in clinical practice and research worldwide. The aim of this paper is to review the literature on the applicability of the GMFCS in Cerebral Palsy. The field of search included search on Bireme of electronic databases Lilacs, Medline, PubMed and SciELO; as well as in books, monographs and related theses. This research was conducted in CADIP (Learning, Documentation, Information and Research Center from FMABC, Santo André). Data from this study may be useful in functional characterization of clinical condition, direction for the choice of assessments and planning of clinical interventions for this population. The GMFCS is a simple tool which is considered essential by the literature because it allows accurate detection of the child motor potential and provides greater efficacy and greater homogeneity in questionnaires.

Keywords: Cerebral palsy; motor skills disorders; motor skills.

Recebido: $1 / 3 / 2010$

Revisado: $14 / 4 / 2010$

Aprovado: 7/6/2010

\footnotetext{
Trabalho realizado na Faculdade de Medicina do ABC (FMABC), Santo André (SP), Brasil

Curso de Fisioterapia da Faculdade de Medicina do ABC (FMABC), Santo André, (SP) Brasil

Endereço para correspondência: Ana Ivone Antonia de Oliveira - Av. Príncipe de Gales, 821 - Príncipe de Gales - Santo André (SP), Brasil - e-mail: annai557@hotmail.com
} 


\section{Introdução}

A paralisia cerebral (PC), denominada encefalopatia crônica não progressiva da infância (ECNPI) ${ }^{1}$, é consequência de uma lesão que pode ocorrer nos períodos pré, peri ou pós-natal afetando o sistema nervoso central em fase de maturação estrutural e funcional ${ }^{2}$. É considerada a causa mais comum de incapacidade física ${ }^{3}$, sendo caracterizada pelo comprometimento neuromotor ${ }^{1,2}$.

Essa patologia é diagnosticada por meio de exame clínico, sendo que raramente é necessário recorrer a exames complementares para sua comprovação. Assim, é importante considerar todos os sinais clínicos junto com sua história e dinâmica ${ }^{4}$.

Nos Estados Unidos, a incidência de PC varia entre 1,5 e $5,9 / 1.000$ nascidos vivos ${ }^{5}$. No Brasil não existem dados estatísticos precisos, estima-se que a cada 1.000 crianças que nascem, sete são portadoras de PC. Nos países em desenvolvimento, esse número pode estar relacionado a problemas gestacionais, condições precárias de nutrição materna e infantil com atendimentos médico e hospitalar muitas vezes inadequados ${ }^{3,5}$.

A PC pode interferir de forma importante na interação da criança em contextos relevantes, influenciando não só a aquisição e o desempenho de marcos motores básicos (rolar, sentar, engatinhar, andar) como também as atividades da rotina diária, como tomar banho, alimentar-se, vestir-se, locomover-se em ambientes variados entre outras ${ }^{2,5}$.

A PC é classificada de acordo com o tipo clínico em atáxico, espástico piramidal, extrapiramidal, hipotônico e misto, e também pela sua distribuição topográfica: hemiparesia (compromete um hemicorpo), diparesia (maior acometimento em membros inferiores) e tetraparesia (acometimento global dos quatros membros) ${ }^{3,6}$.

No decorrer do desenvolvimento e com o avançar da idade, essas crianças passam por mudanças da função motora, sendo de extrema importância avaliar e acompanhar o desenvolvimento das habilidades para que seja possível estabelecer um provável prognóstico ${ }^{2,6}$.

O estabelecimento do prognóstico da função motora grossa nessas crianças é prejudicado pela escassez de sistemas padronizados para classificação, baseados nas habilidades e limitações funcionais ${ }^{7}$.

A primeira versão do Sistema de Classificação da Função Motora Grossa (GMFCS) foi desenvolvido por Palisano et al. ${ }^{1,8}$ no centro de reabilitação CanChild (Center for Childhood Disability Research) do Canadá, com o propósito de atender a necessidade de estabelecer um sistema padronizado para classificar a funcionalidade motora, sendo análogo aos sistemas de estadiamento e graduação utilizados na medicina para descrever o câncer ${ }^{8}$.

Os autores acreditam que essa semelhança de classificação pode facilitar a comunicação entre profissionais e familiares, sua utilização em serviços de reabilitação, a criação de bases de dados e registros e a comparação e generalização de resultados em pesquisas clínicas?

Sua criação foi baseada nas pontuações do método padronizado intitulado Mensuração da Função Motora Grossa (GMFM), com crité- rios funcionais ${ }^{7}$. . Esse método é um sistema de avaliação quantitativo contendo 88 itens, desenvolvido por Russel et al. (1 $1^{\text {a }}$ versão em 1989), com o propósito de mensurar alterações na função motora ampla de crianças com PC?

Esses itens são agrupados em cinco dimensões, sendo A: deitar e rolar; B: sentar; C: engatinhar e ajoelhar; D: ficar em pé; E: andar, correr e pular?. Sua pontuação é obtida pela observação das crianças e graduada em uma escala ordinal de 4 pontos, na qual: $0=$ não realiza; 1 $=$ inicia atividade $; 2$ completa parcialmente a atividade $3=$ completa a atividade.

Já o GMFCS possui cinco níveis de classificação baseados nas habilidades e na iniciativa do movimento, com ênfase particular em sentar e andar? ${ }^{7}$.

Esse instrumento é validado ${ }^{10}$ e possui resultados confiáveis ${ }^{11}$, sendo adotado internacionalmente e com ampla aplicação ${ }^{12}$.

A literatura tem demonstrado atualmente a preferência em classificar as crianças com $\mathrm{PC}^{13}$ de acordo com sua independência funcional nas funções motoras grossas e finas ${ }^{14}$. O GMFCS é aplicado de acordo com a idade da criança e os estudos sustentam o conceito de que essa classificação possui bom grau de estabilidade ao longo dos anos ${ }^{15}$, ou seja, uma criança geralmente permanece no mesmo nível ${ }^{16}$.

Assim, reunir informações sobre as finalidades e resultados de seu emprego na clínica é de grande valia, pois facilita o acesso a informações pelos profissionais envolvidos no tratamento dessas crianças.

Esta revisão da literatura objetivou reunir evidências sobre as possibilidades de aplicação do Sistema de Classificação da Função Motora Grossa (GMFCS) na Paralisia Cerebral, suas vantagens e benefícios.

\section{Métodos}

Este trabalho utilizou materiais disponíveis na biblioteca CADIP (Centro de Aprendizagem, Documentação, Informação e Pesquisa da FMABC, Santo André), bem como nos bancos de dados LILACS Bireme (Base de dados da literatura Latino Americana em Ciências da Saúde), MEDLINE - Índex Medicus (Medical Literature Analysis and Retrieval System Online), SciELO (Scientific Eletronic Library Online) e PubMed (mantido pela National Library of Medicine). Foram selecionados trabalhos com os seguintes descritores: paralisia cerebral, transtornos das habilidades motoras e destreza motora.

Os critérios de inclusão foram: livros, teses, monografias e artigos indexados, todos publicados a partir de 1997 (data da criação do GMFCS) e o de exclusão foi material de fonte não-confiável.

\section{Desenvolvimento}

O desenvolvimento motor da criança com PC restringe a experimentação de padrões normais de movimentos funcionais que são essenciais para o desenvolvimento motor normal ${ }^{17}$. Consequentemente, há diminuição na coordenação e no controle dos movimentos voluntários 
e da postura, ocasionando alterações no desenvolvimento motor ${ }^{18}$ que refletem, muitas vezes, no aparecimento tardio ou, até mesmo, no nãoaparecimento de alguns padrões motores $^{19}$. Sabe-se que esta população apresenta um déficit motor em virtude da lesão cerebral, contudo, percebe-se também, atualmente, uma grande preocupação em inseri-las em programas de atividades motoras ${ }^{20}$. Entretanto, são poucos os trabalhos que utilizam instrumentos de avaliação motora para mensurar o desenvolvimento motor e os benefícios dos programas de atividades motoras nesta população ${ }^{21}$.

O GMFCS é utilizado para mostrar o potencial funcional de crianças com PC, assim como para estabelecer o desenvolvimento esperado em longo prazo $^{17}$. Estudos apresentados na literatura sugerem que essas crianças podem apresentar diferenças significativas na caracterização dos componentes neuromotores, sendo possível que as diferenças não sejam evidentes quando se observa a realização das funções de transferências e de locomoção ${ }^{22}$. Com base nesta afirmação, pode-se concluir que, apesar das alterações nos componentes neuromotores observadas, as crianças acabam desenvolvendo estratégias motoras que lhes permitem desempenhar atividades funcionais ${ }^{23}$. Seu uso exige familiaridade com a criança, mas não é um teste que requeira nenhum treinamento formal 24 .

É uma ferramenta que classifica a capacidade funcional de crianças com PC em cinco níveis. Sua validade e confiabilidade foram comprovadas por profissionais, considerando sua aplicabilidade em crianças de 2 a 12 anos $^{24}$. Os relatos de familiares e profissionais sobre os resultados do uso desta classificação foram considerados excelentes, fato que reafirma a confiabilidade do método para medir a capacidade funcional motora grossa de crianças com PC, o que é particularmente útil em estudos observacionais com populações extensas ${ }^{23}$.

Sua versão expandida e revisada inclui a faixa etária dos 12 aos 18 anos e engloba conceitos inerentes à Classificação Internacional da Funcionalidade, Incapacidade e Saúde (CIF). É enfatizado o desempenho habitual em casa, na escola e na comunidade ${ }^{1,10}$.

Em 2008, Sverdberg et al. analisaram a capacidade funcional motora de crianças com PC usando o GMFCS. Seu estudo analisou relatórios dos pais com informações relevantes para classificar as crianças de acordo com os níveis do GMFCS, nas respectivas idades, e os níveis obtidos confirmaram os resultados encontrados na versão original ${ }^{25}$.

As distinções entre os níveis de função motora são baseadas nas limitações funcionais, na necessidade de tecnologia assistida, incluindo aparelhos auxiliares de locomoção (tais como andadores, muletas e bengalas) e cadeira de rodas, em menor grau, na qualidade de movimento ${ }^{8}$. O Nível I inclui crianças com disfunção neuromotora cujas limitações funcionais são menores do que aquelas normalmente associadas à PC, que tradicionalmente são diagnosticadas com quadro de "disfunção cerebral de severidade mínima”. As distinções entre os Níveis I e II não são tão evidentes quanto as distinções entre os outros níveis, especialmente para crianças com menos de dois anos de idade ${ }^{25}$.
O GMFCS tem sido empregado em pesquisas principalmente para selecionar e descrever amostras de estudos e para superar preconceitos durante o tratamento ${ }^{26}$. Há vários exemplos de resultados de pesquisas que estão sendo exploradas com análises de subgrupos por nível do GMFCS. Isto pode ser particularmente útil na formação de hipóteses para futuras pesquisas sobre intervenções orientadas para as crianças em diferentes níveis do GMFCS, mas com cuidado para não estabelecer conclusões baseadas em estudos com apenas um pequeno número de indivíduos em cada grupo ${ }^{18}$.

Neste contexto, clínicos podem tirar proveito do conhecimento relacionado ao caso clínico das crianças em cada um dos cinco níveis do GMFCS para estabelecer prováveis objetivos terapêuticos. O impacto do GMFCS na prática clínica pode começar durante o estabelecimento do diagnóstico, facilitando a compreensão das famílias a respeito das habilidades atuais da criança, no prognóstico e, posteriormente, no planejamento para intervenção de acordo com as necessidades futuras, assim como , pode auxiliar na definição de objetivos funcionais ${ }^{19}$.

Tieman et al. analisaram a variabilidade na mobilidade de 183 crianças com idade entre seis e 12 anos portadoras de PC nos diferentes níveis de classificação do GMFCS. Para esses autores, o fator contextual da casa, escola e comunidade pode ter impacto importante sobre o desempenho da mobilidade. As características físicas, como as superfícies do solo, tendem a variar de acordo com o ambiente, como na escola, ao ar livre e na comunidade. Seus resultados mostraram que crianças classificadas no mesmo nível funcional apresentaram graus variados de independência em casa, maior restrição na escola e maior prejuízo ainda na comunidade ${ }^{15}$

Um estudo longitudinal de acompanhamento do desenvolvimento da função motora grossa foi realizado em 586 crianças com PC e idades variando entre 1 e 12 anos. Os resultados do estudo mostraram que as pontuações do GMFCS são válidas para a classificação das habilidades motoras e as limitações das crianças com PC, sendo possível estabelecer uma curva de evolução esperada dentro de cada nível ao longo do tempo ${ }^{7}$.

Em 2004, um estudo realizado na Santa Casa de Misericórdia de São Paulo, teve como objetivo classificar uma amostra de 100 crianças com diagnóstico de PC, segundo o GMFCS. Os resultados caracterizaram funcionalmente a população, sendo que 35\% delas pertenciam ao nível I, 12\% ao nível II, 19\% ao nível III, 13\% ao nível IV, 11\% ao nível V e $10 \%$ não puderam ser classificadas por não terem atingido as etapas do desenvolvimento motor normal necessárias ${ }^{4}$.

Vários estudos correlacionaram o GMFCS com o GMFM para analisar essas crianças como: Rosenbaum et al. ${ }^{24}$, Oeffinger et al. ${ }^{26}$, Hägglund et al. ${ }^{27}$, Beckung et al. ${ }^{28}$, Hanna et al. ${ }^{29}$ Ambas as ferramentas são utilizadas amplamente para comparar técnicas e procedimentos clínicos e fisioterapêuticos ${ }^{10}$, e proporcionam aos profissionais e familiares melhor visualização do potencial motor da criança. Porém, cada instrumento tem suas particularidades e são considerados complementares para auxiliar na elaboração das metas terapêuticas ${ }^{30}$. 
Outro estudo analisou um total de 657 crianças com PC, com idade entre 1 e 13 anos ${ }^{24}$ e descreveu os padrões do desenvolvimento motor total. O GMFCS foi aplicado longitudinalmente para criar curvas, traçar taxas e limites da função esperada conforme a gravidade da deficiência motora. Estas curvas possibilitam precisão do prognóstico, com informações disponíveis para as famílias e profissionais da saúde, permitindo monitorar os efeitos de intervenções específicas no decorrer do desenvolvimento motor ${ }^{24}$.

Beckung ${ }^{28}$ et al. Realizaram um estudo de caracterização populacional com 317 crianças entre 1 e 15 anos, dos tipos clínicos espástico, discinético e atáxico e diferentes topografias. Os níveis III e IV foram os mais frequentes, independentemente do tipo de comprometimento ${ }^{27}$. De acordo com os resultados encontrados, os autores sugerem que o GMFCS é útil para o estabelecimento de grupos amostrais homogêneos para estudos clínicos que requerem habilidades motoras funcionais semelhantes ${ }^{26,29}$.

Ao longo do tempo, o GMFCS se mostrou uma metodologia de avaliação estável, sem necessidade de treinamento específico, podendo ser aplicado em aproximadamente cinco minutos ${ }^{31}$. Não requer tempo excessivo porque parte do princípio de que crianças com a função motora total próxima do normal são classificadas como nível I e crianças sem controle cervical e dependentes para todos os aspectos dos seus cuidados pessoais são categorizadas como nível $\mathrm{V}^{32}$.

\section{Considerações finais}

O GMFCS descreve a função motora grossa em relação às atividades funcionais, com ênfase na independência alcançada nas habilidades motoras sentar e andar com base no conceito de deficiência e de limitação funcional global de crianças com PC.

Os dados deste estudo podem ser úteis na caracterização funcional do quadro clínico, no direcionamento para escolha de avaliações e no planejamento das intervenções clínicas a essa população. O GMFCS é uma ferramenta simples, porém, considerada essencial pela literatura consultada, pois ao permitir a detecção exata do potencial motor da criança possibilita maior eficácia terapêutica e maior homogeneidade nas pesquisas.

\section{Referências}

1. Ozu MHU, Galvão MCS. Fisioterapia na paralisia cerebral. In: Borges D, Moura EW, Lima E, Silva PAC. Fisioterapia: aspectos clínicos e práticos da reabilitação. São Paulo: Artes Médicas; 2007. p. 13-25.

2. Marinho, APS, Souza MAB, Pimentel AM. Desempenho funcional de crianças com paralisia cerebral diparéticas e hemiparéicas. Rev Ciênc Méd Biol. Salvador. 2008;7(1):57-66.

3. Christofoletti G, Hygashi F, Godoy ALR. Paralisia cerebral: uma análise do comprometimento motor sobre a qualidade de vida. Fisioter Mov Curitiba. 2007;20(1):37-44.

4. Lanfredi APA, Narvaes S, Peres PT, Matta DSP. Nível de habilidade motora funcional em crianças com paralisia cerebral. Reabil. 2004,25(6):20-3.

5. Mancini MC, Alves ACM, Schaper C, Figueiredo EM, Sampaio RF, Coelho ZAC, Tirado MGA. Gravidade da paralisia cerebral e desempenho funcional. Rev Bras Fisioter. 2004,8(3):253-60

6. Camargos ACR, Fontes PLB, Gontijo EG, Araújo FM, Cota K, et al. Fisioterapia associada à toxina botulínica na diplegia espástica: um relato de caso. Fisioter Mov Curitiba. 2007;20(3):17-24.

7. Palisano RJ, Hanna SE, Rosenbaum PL. Validation of a model of gross motor function for children with cerebral palsy. Phys Ther. 2000;80(10):974-85.

8. Palisano R, Rosenbaum P, Stephen W, Russel D, Wood E, Galapi B. Development and reliability of a system, to classify gross motor function in children with cerebral palsy. Dev Med Child Neurol. 1997;39(4):214-23.

9. Pina LV, Loureiro APC. 0 GMFM e sua aplicação na avaliação motora de crianças com paralisia cerebral. Fisiot Mov Curitiba. 2006;19(2):91-100.

10. Andrada MG, Virella D, Calado E, Gouveia R, Alvarelhão J, Folha T. Versão
Portuguesa - Sistema de classificação da função motora global (SCFMG). 2007. [citado 14 mai. 2010] Disponível em: < www.canchild.ca ou www.fappc.pta> .

11. Mascarenhas T. Análise das escalas desenvolvidas para avaliar a função motora de pacientes com paralisia cerebral. [Dissertação]. Faculdade de Ciências Médicas da Saúde (SP): 2008.

12. Carnahan $\mathrm{KD}$, Arner $\mathrm{M}$, Hägglund $\mathrm{G}$. Association between gross motor function (GMFCS) and manual ability (MACS) in children with cerebral palsy. A population-based study of 359 children. BMC Musculoskelet Disord. 2007:8(50):1-7.

13. Francischetti SSR. A sobrecarga em cuidadores familiares de crianças portadoras de paralisia cerebral grave. [Tese]. Universidade Presbiteriana Mackenzie. São Paulo (SP): 2006.

14. Cury VCR, Mancini MC, Melo AP, Fonseca ST, Sampaio RF, Tirado MGA, et al. Efeitos do uso de órtese na mobilidade funcional de crianças com paralisia cerebral. Rev Bras Fisioter. 2006;10(1):67-74.

15. Tieman B, Palisano RJ, Gracely EJ, Rosenbaum PL. Variability in Mobility of Children with Cerebral Palsy. Pediatric Phys Ther. 2007;19(3):180-7 .

16. Chagas PSC, Defilipo EC, Lemos RA, Mancini MC, Frônio JS, Carvalho RM. Classificação da função motora e do desempenho funcional de crianças com paralisia cerebral. Rev Bras Fisioter. 2008;12(5):409-16.

17. Mancini MC, Teixeira S, Araújo LG, Paixão ML, Magalhães LC, Coelho ZAC, et al. Estudo do desenvolvimento da função motora aos 8 e 12 meses de idade em crianças nascidas pré-termo e a termo. Arq Neuropsiquiatr. 2002;60(4):974-80.

18. Morris C. Gross motor function classification system: impact and utility. Dev Med Child Neurol. 2004;46(1):60-5. 
19. Gorter JW, Ketelaar M, Rosenbaum P, Helders PJM, Palisano R. Use of the GMFCS in infants with CP: the need for reclassification at age 2 years or older. Dev Med Child Neurol. 2008;51(1):46-52.

20. Wood E, Rosenbaum P. The gross motor function classification system for cerebral palsy: a study of reliability and stability over time. Dev Med Child Neurol. 2000;42(5):292-6.

21. Rosa GKB, Marques I, Medina-Paps J, Gobbi LTB. Desenvolvimento motor de criança com paralisia cerebral: avaliação e intervenção. Rev Bras Ed Esp. 2008;14(2):163-76.

22. Palisano RJ, Tieman BL, Walter SD, Bartlett DJ, Rosenbaum PL, Russell D, et al. Effect of environmental setting on mobility methods of children with cerebral palsy. Dev Med Child Neurol. 2003;45(2):113-20.

23. Vohr BR, Msall ME, Wilson D, Wright LL, McDonald S, Poole WK. Spectrum of Gross Motor Function in Extremely Low Birth Weight Children With Cerebral Palsy at 18 Months of Age. Pediatrics. 2005;116(1):123-9.

24. Rosenbaum PL, Walter SD, Hanna SE, Palisano RJ, Russell DJ, Raina P, et al. Prognosis for Gross Motor Function in Cerebral Palsy: Creation of Motor Development Curves. JAMA. 2002;288(11):1357-63.

25. Svedberg LE, Englund E, Malker H, Stener-Victorin E. Parental perception of cold extremities and other accompanying symptoms in children with cerebral palsy. Eur Jour of Paediatric Neurol. 2008;12(2):89-96 .
26. Oeffinger DJ, Tylkowski CM, Rayens MK, Davis RF, Gorton GE, Nicholson $\mathrm{DE}$, et al. Gross motor function classification system and outcome tools for assessing ambulatory cerebral palsy: a multicenter study. Dev Med Child Neurol. 2004;46(5):311-9.

27. Hägglund $G$, Lauge-Pedersen $H$, Wagner $P$. Characteristics of children with hip displacement in cerebral palsy. BMC Musculoskeletal Disorders. 2007;8(101):1-6.

28. Beckung E, Carlsson G, Carlsdotter S, Uvebrant P. The natural history of gross motor development in children with cerebral palsy aged 1 to 15 years. Dev Med Child Neurol. 2007;49(10):751-6.

29. Hanna SE, Bartlett DJ, Rivard LM, Russell DJ. Reference Curves for the Gross Motor Function Measure: Percentiles for Clinical Description and Tracking Over Time Among Children With Cerebral Palsy. Phys Ther 2008;88(5):596-607.

30. Morris C, Kurinczuk JJ, Fitzpatrick R, Rosenbaum PL. Reliability of the manual ability classification system for children with cerebral palsy. Dev Med Child Neurol. 2006;48(12):950-3.

31. Peralta-Carcelen M, Marybeth Moses, Adams-Chapman I, Gantz M, Vohr BR. Stability of Neuromotor Outcomes at 18 and 30 Months of Age After Extremely Low Birth Weight Status. Pediatrics. 2009;123(5):887-95.

32. Palisano RJ, Copeland WP, Galuppi BE. Performance of physical activities by adolescents with cerebral palsy. Phys Ter. 2007;87(1):77-87. 